\title{
Research on Mobile Cloud Robotics based on Cloud Computing
}

\author{
Xinqiang $\mathrm{MA}^{\mathrm{a}}$, Yi HUANG ${ }^{\mathrm{b}^{*}}$ \\ Key Laboratory of Machine Vision and Intelligent Information System, Chongqing University of Arts \\ and Sciences, Chongqing, 402160, China \\ aemail: xinqma@163.com, ${ }^{\mathrm{b} *}$ corresponding author email: cqhy@21cn.com
}

Keywords: Cloud Computing; Cloud Robotics; Mobile Cloud Computing; Mobile Cloud Robotics Abstract. Cloud Computing has been envisioned as the next-generation architecture of IT Enterprise. Together with an explosive growth of the mobile applications and emerging of cloud computing concept, mobile cloud computing has been introduced to be a potential technology for mobile services. Cloud robotics is a paradigm where robots offload their heavy computations and storage needs to the cloud while focusing on simpler computation tasks. This work studies a mobile cloud robotics architecture that combines these mobile cloud computing and cloud robotics.

\section{Introduction}

Cloud computing has become a ubiquitous term used today for computational models where tasks are executed by several interconnected computers offering greater capabilities in terms of processing power and storage than standalone solutions. Powerful computing infrastructures hidden in virtualization software make systems to be like a true physical computer, but with the flexible specification of details such as number of processors, memory and disk size, and operating system. The use of these virtual computers is known as Cloud Computing [1,2].Researchers have applied the principles of cloud computing to the field of robotics as well as to mobile environments resulting in the emergence of topics such as cloud robotics and mobile cloud[3].

Google's James Kuffner coined the term "Cloud Robotics" in 2010. Cloud Robot and Automation systems can be broadly defined as any robot or automation system that relies on data or code from a network to support its operation, i.e., where not all sensing, computation, and memory is integrated into a single standalone system. Cloud robotics is a field of robotics that attempts to invoke cloud technologies such as cloud computing, cloud storage, and other Internet technologies centered on the benefits of converged infrastructure and shared services for robotics. When connected to the cloud, robots can benefit from the powerful computational, storage, and communications resources of modern data centre in the cloud, which can process and share information from various robots or agent (other machines, smart objects, humans, etc.). It is possible to build lightweight, low cost, smarter robots have intelligent "brain" in the cloud. The "brain" consists of data center, knowledge base, task planners, deep learning, information processing, environment models, communication support etc[4,5].The concept of mobile cloud computing (MCC) relies on using mobile devices such as smart phones, tablets, and embedded devices to detect phenomena, while offloading the heavy computation and storage needs to the cloud. MCC integrates the cloud computing into the mobile environment and overcomes obstacles related to the performance (e.g., battery life, storage, and bandwidth), environment (e.g., heterogeneity, scalability, and availability), and security (e.g., reliability and privacy) discussed in mobile computing[6].

In this paper we propose a mobile cloud robotics architecture that will integrate concepts from cloud robotics and mobile cloud.

\section{Cloud computing}

Cloud computing represents one of the most significant shifts in information technology many of us are likely to see in our lifetimes. It offers an innovative business model for organizations to adopt IT services without upfront investment. According to Gartner's Hype cycle, cloud computing has reache 
-d a maturity that leads it into a productive phase. This means that most of the main issues with cloud computing have been addressed to a degree that clouds have become interesting for full commercial exploitation. Cloud computing frameworks (see Fig. 1) metaphor: For a user, the network elements representing the provider-rendered services are invisible, as if obscured by a cloud. The goal of cloud com- puting is to allow users to take benefit from all of these technologies, without the need for deep knowledge about or expertise with each one of them. The cloud aims to cut costs, and helps the users focus on their core business instead of being impeded by IT obstacles. Cloud computing providers offer their services according to several fundamental models [2]: infrastructure-, platform- and software-as-a-service.

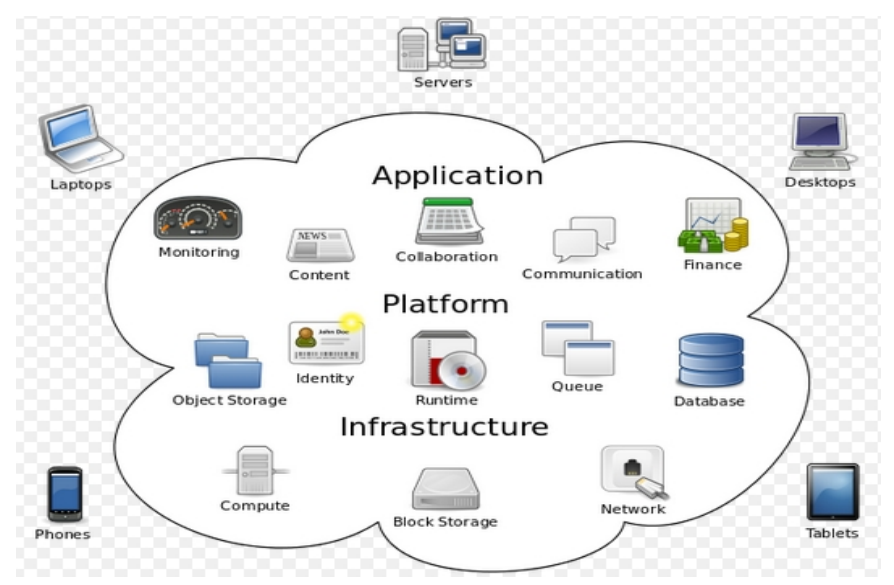

Fig.1. Cloud computing frameworks

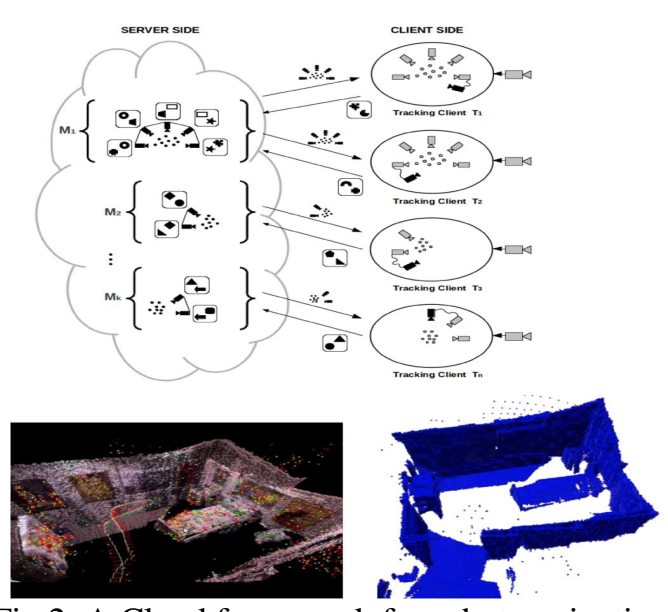

Fig.2. A Cloud framework for robot navigation

It is important to acknowledge that the Cloud is prone to varying network latency and quality of service.Cloud Computing has potential to speed up many computationally-intensive robotics and automation systems applications such as robot navigation by performing SLAM in the Cloud [7], as illustrated in Fig. 2 and next-view planning for object recognition [8].

\section{Mobile cloud computing}

The Mobile cloud computing forum defines MCC as follows [9]: 'Mobile cloud computing at its simplest, refers to an infrastructure where both the data storage and data processing happen outside of the mobile device. Mobile cloud applications move the computing power and data storage away from mobile phones and into the cloud, bringing applications and mobile cloud to not just smartphone users but a much broader range of mobile subscribers'. MCC provides mobile users with the data process-ing and storage services in clouds. The mobile devices do not need a powerful configuration (e.g., CPU speed and memory capacity) because all the complicated computing modules can be processed in the clouds.

From the concept of MCC, the general architecture of MCC can be shown in Fig. 3[6]. In Figure 3, mobile devices are connected to the mobile networks via base stations (e.g., base transceiver station, access point, or satellite) that establish and control the connections (air links) and functional interfaces between the networks and mobile devices. Mobile users' requests and information (e.g., ID and location) are transmitted to the central processors that are connected to servers providing mobile network services. Here, mobile network operators can provide services to mobile users as authentication, authorization, and accounting based on the home agent and subscribers' data stored in databases. After that, the subscribers' requests are delivered to a cloud through the Internet. In the cloud, cloud controllers process the requests to provide mobile users with the corresponding cloud services. These services are developed with the concepts of utility computing, virtualization, and service-oriented architecture (e.g., web, application, and database servers). This architecture is commonly used to demonstrate the effectiveness of the Cloud computing model in terms of meeting the user's requirements. Cloud computing is known to be a promising solution for Mobile computing because of many reasons (e.g., mobility, communication, and portability [10]). 


\section{Cloud Robotics}

As illustrated in Fig.4, the Cloud has potential to enhance a broad range of robots and automation systems. Cloud Robot and Automation systems can be broadly defined as follows: Any robot or automation system that relies on either data or code from a network to support its operation, i.e., where not all sensing, computation, and memory is integrated into a single standalone system. This definition is intended to include future systems and many existing systems that involve networked teleoperation or networked groups of mobile robots[4,11].

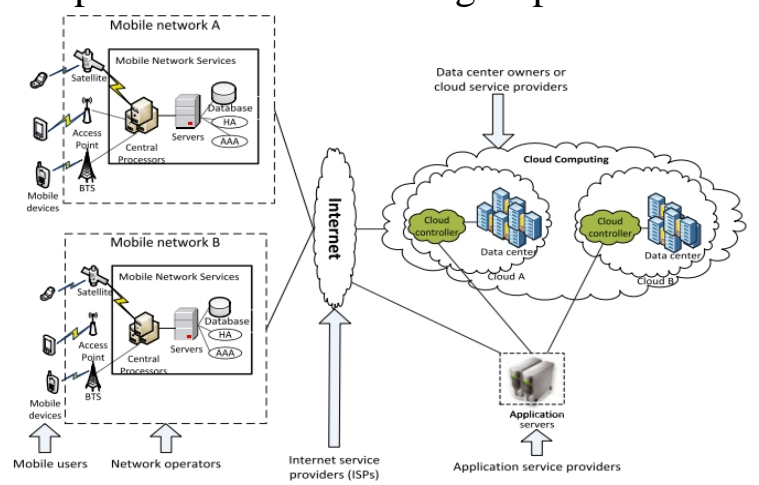

Fig. 3. Mobile cloud computing architecture.

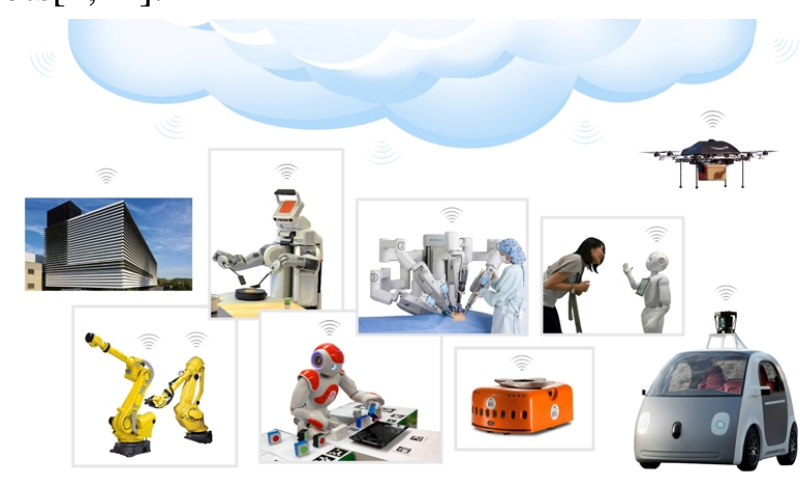

Fig. 4. Based-cloud of robots and automation systems

Using the Cloud for robotics and automation systems introduces many new challenges[12]. The connectivity inherent in the Cloud raises a range of privacy and security concerns. Many of these regulations mandate particular controls (such as strong access controls [13] and audit trails) and require regular reporting. Cloud customers must ensure that their cloud providers adequately fulfill such requirements as appropriate, enabling them to comply with their obligations since, to a large extent, they remain accountable.

\section{Mobile Cloud Robotics}

A mobile robot is an automatic machine that is capable of locomotion. Mobile robots have the capability to move around in their environment and are not fixed to one physical location. Mobile robots can be "autonomous" which means they are capable of navigating an uncontrolled environment without the need for physical or electro-mechanical guidance devices. Alternatively, mobile robots can rely on guidance devices that allow them to travel a pre-defined navigation route in relatively controlled space (AGV - autonomous guided vehicle). By contrast, industrial robots are usually more-or -less stationary, consisting of a jointed arm (multi-linked manipulator) and gripper assembly (or end effector), attached to a fixed surface. Mobile robots have become more commonplace in commercial and industrial settings. Hospitals have been using autonomous mobile robots to move materials for many years. Mobile robots are also a major focus of current research and almost every major university has one or more labs that focus on mobile robot research. Mobile robots are also found in industrial, military and security settings. Domestic robots are consumer products, including entertainment robots and those that perform certain household tasks such as vacuuming or gardening[14].

JMR Álamo et al. proposed a mobile cloud robotics architecture that will integrate concepts from cloud robotics and mobile cloud. For practical and pedagogical purposes they designed the architecture to work with the popular and relatively inexpensive Lego NXT robot(see Fig.5 and Fig.6). The idea is to allow the robots to communicate among themselves and also to connect to a cloud computing platform[4].

\section{Conclusions}

Mobile cloud Robotics is one of the mobile technology trends in the future because it combines the advantages of both mobile cloud computing and cloud robotics, thereby providing optimal services for mobile users and robots. Using the Cloud for mobile robotics systems introduces many new challenges. Such as security and privacy. Future work includes finishing the implementation of some features of the 
architecture, debugging, and more testing.
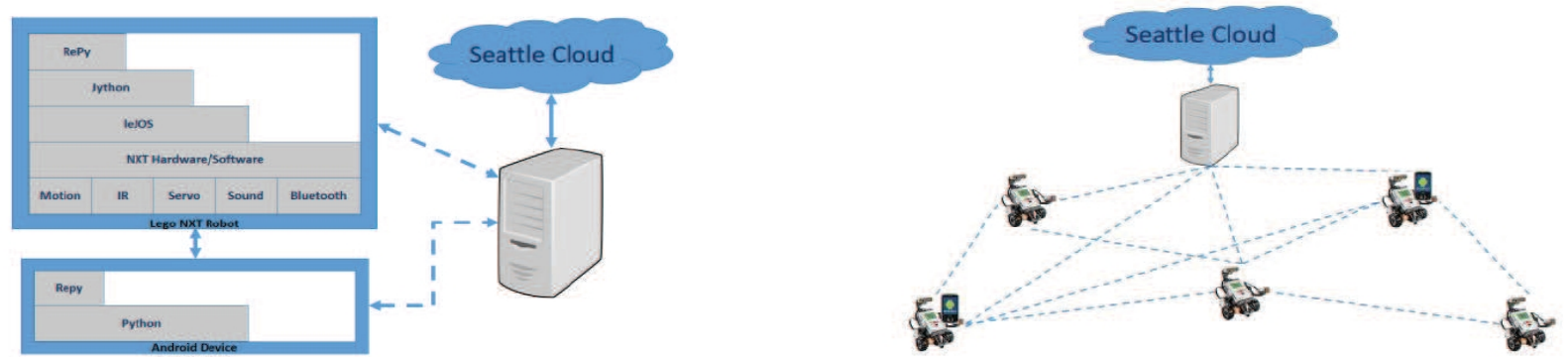

Fig.5. Hybrid Lego NXT Mobile Cloud Robotics Architecture Fig.6. Network of Robots for Hybrid Lego NXT

\section{Acknowledgements}

This work was financially supported by the Natural Science Foundation of CQ CSTC (cstc2015jcyjA40026, cstc2014jcyjA40056 and cstc2013jcyjA40053), Scientific and Technological Research Program of Chongqing Municipal Education Commission (KJ1401112, KJ111218, KJ1401117 and KJ1501134) and Natural Science Foundation of Ycstc(2014bf2001).

\section{References}

[1] Borko Furht, Armando Escalante, Handbook of Cloud Computing, Springer, (2011)

[2] http://en.wikipedia.org/wiki/Cloud_computing\#cite_note-90, January, (2016)

[4] JMR Álamo, B Mendoza, A Carranza. Towards an Aarchitecture for Mobile Cloud Robotics. IHART - Volume 31, (2013)

[4] Ben Kehoe, Sachin Patil, Pieter Abbeel, Ken Goldberg. A Survey of Research on Cloud Robotics and Automation. IEEE Transactions on Automation Science and Engineering (T-ASE): Special Issue on Cloud Robotics and Automation. Vol. 12, no. 2. Apr. (2015)

[5] https://en.wikipedia.org/wiki/Cloud_robotics\#cite_note-RASrobot-1,January, (2016)

[6] Dinh, H. T., Lee, C., Niyato, D., \& Wang, P. A survey of mobile cloud computing: architecture, applications, and approaches. Wireless Communications and Mobile Computing, n/a-n/a. doi:10.1002/wcm.1203,(2011)

[7] E. Plaku, K. E. Bekris, B. Y. Chen, A. M. Ladd, and L. E. Kavraki, Sampling-based Roadmap of Trees for Parallel Motion Planning, IEEE Transactions on Robotics (T-RO), vol. 21, no. 4, pp. 597-608,(2005)

[8] L. Riazuelo, J. Civera, and J. Montiel, "C2TAM: A Cloud Framework for Cooperative Tracking and Mapping," Robotics and Autonomous Systems, vol. 62, no. 4, pp. 401-413, (2013)

[9]L. Turnbull and B. Samanta, "Cloud robotics: Formation control of a multi robot system utilizing cloud infrastructure," in Proceedings of IEEE Southeastcon. IEEE, (2013), pp. 1-4

[10]http://www.mobilecloudcomputingforum.com/, January, (2016)

[11]Forman GH, Zahorjan J. The Challenges of mobile computing. IEEE Computer Society Magazine (1994),27(4): 38-47.

[12]http://goldberg.berkeley.edu/cloud-robotics/, January, (2016)

[13] Yi. Huang and Xinqiang Ma. An access control model based on Trusted Computing, Journal of Chongqing University of Arts and Sciences, 29(3) 54-57, (2010)

[14] https://en.wikipedia.org/wiki/Mobile_robot, January, (2016) 